PROCEEDINGS OF THE

AMERICAN MATHEMATICAL SOCIETY

Volume 132, Number 8, Pages 2215-2220

S 0002-9939(04)07322-8

Article electronically published on March 10, 2004

\title{
FALTINGS' THEOREM FOR THE ANNIHILATION OF LOCAL COHOMOLOGY MODULES OVER A GORENSTEIN RING
}

\author{
K. KHASHYARMANESH AND SH. SALARIAN
}

(Communicated by Bernd Ulrich)

\begin{abstract}
In this paper we study the Annihilator Theorem and the Localglobal Principle for the annihilation of local cohomology modules over a (not necessarily finite-dimensional) Noetherian Gorenstein ring.
\end{abstract}

\section{INTRODUCTION}

Let $A$ be a commutative Noetherian ring, let $\mathfrak{a}$ and $\mathfrak{b}$ be ideals of $A$ and let $M$ be a finitely generated $A$-module. We first briefly recall the invariant $\lambda_{\mathfrak{a}}^{\mathfrak{b}}(M)$ defined in 3 .

By the terminology of [3], the $\mathfrak{b}$-minimum $\mathfrak{a}$-adjusted depth $\lambda_{\mathfrak{a}}^{\mathfrak{b}}(M)$ of $M$ is defined by

$$
\lambda_{\mathfrak{a}}^{\mathfrak{b}}(M)=\inf \left\{\operatorname{depth} M_{\mathfrak{p}}+\operatorname{ht}(\mathfrak{a}+\mathfrak{p}) / \mathfrak{p}: \mathfrak{p} \in \operatorname{Spec}(A) \backslash V(\mathfrak{b})\right\}
$$

where $V(\mathfrak{b})$ denotes the set of prime ideals containing $\mathfrak{b}$.

We shall say that the Annihilator Theorem (for local cohomology modules) holds over $A$ if $\lambda_{\mathfrak{a}}^{\mathfrak{b}}(M)=f_{\mathfrak{a}}^{\mathfrak{b}}(M)$ for every choice of the finitely generated $A$-module $M$ and for every choice of the ideals $\mathfrak{b}, \mathfrak{a}$ of $A$ where $f_{\mathfrak{a}}^{\mathfrak{b}}(M)$ is the $\mathfrak{b}$-finiteness dimension of $M$ relative to $\mathfrak{a}$. Also, we say that the Local-global Principle (for the annihilation of local cohomology modules) holds over $A$ if

$$
f_{\mathfrak{a}}^{\mathfrak{b}}(M)=\inf \left\{f_{\mathfrak{a} A_{\mathfrak{p}}}^{\mathfrak{b} A_{\mathfrak{p}}}\left(M_{\mathfrak{p}}\right): \mathfrak{p} \in \operatorname{Spec}(A)\right\}
$$

for every choice of ideals $\mathfrak{a}, \mathfrak{b}$ of $A$ and every choice of the finitely generated $A$ module $M$.

Faltings' Annihilator Theorem [5] states that if $A$ is a homomorphic image of a regular ring or $A$ has a dualizing complex, then the annihilator theorem (for local cohomology modules) holds over $A$. In [7], Raghavan deduced from Faltings' Annihilator Theorem [5] that if $A$ is a homomorphic image of a regular ring, then the Local-global Principle (for the annihilation of local cohomology modules) holds over $A$. Recently, in [2], Brodmann, Rotthaus and Sharp showed, for every nonnegative integer $r$, that if $A$ is universally catenary and all formal fibres of all localizations of $A$ satisfy Serre's Condition $\left(S_{r}\right)$, then the Annihilator Theorem (for

Received by the editors June 5, 2002 and, in revised form, March 5, 2003.

2000 Mathematics Subject Classification. Primary 13D45, 13E05, 13H10, 13D05, 13C15.

Key words and phrases. Local cohomology modules, Gorenstein rings, annihilator theorem.

This research was in part supported by a grant from IPM (No. 81130021 and No. 81130117). 
local cohomology modules) holds at level $r$ over $A$ if and only if the Local-global Principle (for the annihilation of local cohomology modules) holds at level $r$ over $A$. They also established the Local-global Principle (for the annihilation of local cohomology modules) over an arbitrary commutative Noetherian ring of dimension not exceeding 4.

In this paper, we show that the Local-global Principle and the Annihilator Theorem (for local cohomology modules) hold over a (homomorphic image of) a commutative Noetherian Gorenstein ring. The proof of the main theorem relies heavily on ideas in Brodmann's proof of [1, Satz 3.12] and in Raghavan's proof of [8, 3.1]. But our method is based on the theory of Gorenstein dimension or G-dimension. To the best of our knowledge, this is the first application of this theory in the study of Faltings' Annihilator Theorem.

Throughout the paper, $A$ will denote a commutative Noetherian ring (with nonzero identity), $\mathfrak{a}$, and $\mathfrak{b}$ will denote ideals of $A$, and $M$ will denote a finitely generated $A$-module. We use $\mathbb{N}$ and $\mathbb{N}_{0}$ to denote the set of positive and nonnegative integers respectively. For any unexplained notation and terminology concerning G-dimension we refer the reader to [4].

\section{Results}

Let us, firstly, introduce $\mathfrak{b}$-finiteness dimension of $M$ relative to $\mathfrak{a}$ (see [3] 9.1.5]):

$$
f_{\mathfrak{a}}^{\mathfrak{b}}(M)=\inf \left\{i \in \mathbb{N}_{0}: \mathfrak{b}^{n} H_{\mathfrak{a}}^{i}(M) \neq 0 \text { for all } n \in \mathbb{N}\right\},
$$

where, by convention, the infimum of the empty set of integers is interpreted by $\infty$. Note that if $\mathfrak{a}=\mathfrak{b}$, then $f_{\mathfrak{b}}^{\mathfrak{b}}(M)=f_{\mathfrak{a}}(M)$ is the finiteness dimension of $M$ relative to $\mathfrak{a}$.

We begin by the following remark.

Remark 2.1. It follows from [2, 3.7] that $f_{\mathfrak{a}}^{\mathfrak{b}}(M)=f_{\mathfrak{a}+\mathfrak{b}}^{\mathfrak{b}}(M)$. So one can reduce the study of the Local-global Principle (for the annihilation of local cohomology modules) for arbitrary ideals $\mathfrak{b}$ and $\mathfrak{a}$ to the study of this principle in the case that $\mathfrak{b} \subseteq \mathfrak{a}$.

We now prove some preliminary lemmas and a proposition which help us to conclude the main theorem.

Lemma 2.2. Let $\mathfrak{a}$ and $\mathfrak{b}$ be ideals of $A$ such that $\mathfrak{b} \subseteq \mathfrak{a}$ and $0:{ }_{A} M / \Gamma_{\mathfrak{b}}(M) \subseteq \mathfrak{a}$. Let $s$ be a positive integer. Suppose that $f_{\mathfrak{a} A_{\mathfrak{p}}}^{\mathfrak{b} A_{\mathfrak{p}}}\left(M_{\mathfrak{p}}\right)>s$ for every prime ideal $\mathfrak{p}$ of A. Then $s<\mathrm{ht}_{M} \mathfrak{a}$.

Proof. We suppose that $s \geq \mathrm{ht}_{\mathrm{M}} \mathfrak{a}$, and look for a contradiction. Firstly, we can use the exact sequence

$$
0 \longrightarrow \Gamma_{\mathfrak{b}}(M) \longrightarrow M \longrightarrow M / \Gamma_{\mathfrak{b}}(M) \longrightarrow 0
$$

to deduce the long exact sequence

$$
\cdots \longrightarrow H_{\mathfrak{a}}^{i}\left(\Gamma_{\mathfrak{b}}(M)\right) \longrightarrow H_{\mathfrak{a}}^{i}(M) \longrightarrow H_{\mathfrak{a}}^{i}\left(M / \Gamma_{\mathfrak{b}}(M)\right) \longrightarrow \cdots .
$$

Now, it follows from the assumption that $f_{\mathfrak{a} A_{\mathfrak{p}}}^{\mathfrak{b} A_{\mathfrak{p}}}\left(M / \Gamma_{\mathfrak{b}}(M)\right)_{\mathfrak{p}}>s$ for all $\mathfrak{p} \in \operatorname{Spec}(A)$. Since $\mathrm{ht}_{M / \Gamma_{\mathfrak{b}}(M)} \mathfrak{a} \leq \mathrm{ht}_{M} \mathfrak{a}$, we can thus replace $M$ by $M / \Gamma_{\mathfrak{b}}(M)$ and assume, in our search for a contradiction, that there exists $x \in \mathfrak{b}$ that is a non-zero-divisor on $M$. Since $0::_{A} M \subseteq \mathfrak{a}$, there exists a minimal prime ideal $\mathfrak{p}$ over $\mathfrak{a}$ such that 
$\operatorname{ht}_{M} \mathfrak{p} \leq s$. Set $t:=\operatorname{ht}_{M} \mathfrak{p}$. Then, by [3, 6.1.4], $H_{\mathfrak{a} A_{\mathfrak{p}}}^{t}\left(M_{\mathfrak{p}}\right) \neq 0$ and by hypothesis, $x^{n(\mathfrak{p})} H_{\mathfrak{a} A_{\mathfrak{p}}}^{t}\left(M_{\mathfrak{p}}\right)=0$ for some $n(\mathfrak{p}) \in \mathbb{N}$. Now, using the exact sequence

$$
0 \longrightarrow M \stackrel{x^{n(\mathfrak{p})}}{\longrightarrow} M \longrightarrow \frac{M}{x^{n(\mathfrak{p})} M} \longrightarrow 0,
$$

we obtain the exact sequence

$$
H_{\mathfrak{a} A_{\mathfrak{p}}}^{t}\left(M_{\mathfrak{p}}\right) \stackrel{x^{n(\mathfrak{p})}}{\longrightarrow} H_{\mathfrak{a} A_{\mathfrak{p}}}^{t}\left(M_{\mathfrak{p}}\right) \longrightarrow 0
$$

which, in turn, yields $H_{\mathfrak{a} A_{\mathfrak{p}}}^{t}\left(M_{\mathfrak{p}}\right)=0$; this is the required contradiction.

Lemma 2.3. Let $\mathfrak{a}$ and $\mathfrak{b}$ be ideals of $A$, and let $M$ be a finitely generated A-module. Let $\mathfrak{c}$ be an ideal of $A$ such that $\mathfrak{c} \subseteq 0::_{A} M / \Gamma_{\mathfrak{b}}(M)$. Then $f_{\mathfrak{a}+\mathfrak{c}}^{\mathfrak{b}}(M)=f_{\mathfrak{a}}^{\mathfrak{b}}(M)$.

Proof. Let $n$ be a positive integer such that $\Gamma_{\mathfrak{b}}(M)=0:_{M} \mathfrak{b}^{n}$. Hence, it follows from our assumption that $\mathfrak{b}^{n} r H_{\mathfrak{a}}^{i}(M)=0$, for all $r \in \mathfrak{c}$ and $i \geq 0$, and so $\mathfrak{b}^{n}\left(H_{\mathfrak{a}}^{i}(M)\right)_{r}=0$. Now, the claim follows from the exact sequence

$$
\cdots \longrightarrow H_{\mathfrak{a}+A r}^{i}(M) \longrightarrow H_{\mathfrak{a}}^{i}(M) \longrightarrow\left(H_{\mathfrak{a}}^{i}(M)\right)_{r} \longrightarrow H_{\mathfrak{a}+A r}^{i+1}(M) \longrightarrow \cdots
$$

For an $A$-module $M$ the biduality map is the canonical map

$$
\delta_{M}: M \longrightarrow \operatorname{Hom}_{A}\left(\operatorname{Hom}_{A}(M, A), A\right),
$$

defined by $\delta_{M}(x)(\psi)=\psi(x)$ for $\psi \in \operatorname{Hom}_{A}(M, A)$ and $x \in M$. It is a homomorphism of $A$-modules.

Definition 2.4. [4, 1.1.2] A finitely generated $A$-module $M$ belongs to the G-class $G(A)$ if and only if

(1) $\operatorname{Ext}_{A}^{t}(M, A)=0$ for $t>0$;

(2) $\operatorname{Ext}_{A}^{t}\left(\operatorname{Hom}_{A}(M, A), A\right)=0$ for $t>0$; and

(3) the biduality map $\delta_{M}: M \longrightarrow \operatorname{Hom}_{A}\left(\operatorname{Hom}_{A}(M, A), A\right)$ is an isomorphism.

Definition 2.5. [4, 1.2.1] A G-resolution of a finitely generated $A$-module $M$ is a sequence of modules in $G(A)$,

$$
\cdots \longrightarrow G_{l} \longrightarrow G_{l-1} \longrightarrow \cdots \longrightarrow G_{1} \longrightarrow G_{0} \longrightarrow 0
$$

which is exact at $G_{l}$ for $l>0$ and has $G_{0} / \operatorname{Im}\left(G_{1} \longrightarrow G_{0}\right) \cong M$. That is, there is an exact sequence

$$
\cdots \longrightarrow G_{l} \longrightarrow G_{l-1} \longrightarrow \cdots \longrightarrow G_{1} \longrightarrow G_{0} \longrightarrow M \longrightarrow 0 .
$$

The resolution is said to be of length $n$ if $G_{n} \neq 0$ and $G_{l}=0$ for $l>n$.

Note that every finitely generated $A$-module has a resolution by finitely generated free modules and, thereby, a G-resolution.

Definition 2.6. 4, 1.2.3] A finitely generated $A$-module $M$ is said to have finite G-dimension if it has a G-resolution of finite length. We set $G-\operatorname{dim}_{A} 0=-\infty$, and for $M \neq 0$ we define the G-dimension of $M$ as follows: For any positive integer $n$ we say that $M$ has G-dimension at most $n$, and write $G-\operatorname{dim}_{A} M \leq n$ for short, if and only if $M$ has a G-resolution of length $n$. If $M$ has no G-resolution of finite length, then we say that it has infinite G-dimension and write $G-\operatorname{dim}_{A} M=\infty$.

Remark 2.7. By [4 1.4.9], a commutative Noetherian local ring $A$ is Gorenstein if and only if every finite $A$-module has a finite G-resolution. 
Proposition 2.8. Let $M$ be a finitely generated $A$-module and let $G-\operatorname{dim}_{A} M=0$. Then $\operatorname{grade}(\mathfrak{a}, M) \geq \operatorname{grade}(\mathfrak{a}, A)$, for every ideal $\mathfrak{a}$ of $A$.

Proof. If $\mathfrak{a} M=M$, then grade $(\mathfrak{a}, M)=\infty$ and the result is clear. We therefore suppose that $\mathfrak{a} M \neq M$. Set $g:=\operatorname{grade}(\mathfrak{a}, A)$. We argue by induction on $g$. When $g=0$ the result is clear. We therefore suppose inductively that $g>0$ and that the result has been proved for smaller values of $g$. Since $G-\operatorname{dim}_{A} M=0$, by [4] 1.1.8], we have that $\operatorname{Ass} M \subseteq \operatorname{Ass} A$. Hence there exists $x \in \mathfrak{a}$ such that $x$ is an $M$ and $A$-sequence. Also, by [4, 1.3.6], $G-\operatorname{dim}_{A / x A} M / x M=0$. Note that by our assumption $x M \neq M$. Therefore, by the inductive hypotheses, grade $(\mathfrak{a}, M / x M) \geq$ $\operatorname{grade}(\mathfrak{a}, A / x A)$ and so $\operatorname{grade}(\mathfrak{a}, M) \geq \operatorname{grade}(\mathfrak{a}, A)$.

Lemma 2.9. Let $M$ be a finitely generated $A$-module, and let $\mathfrak{p} \in \operatorname{Spec}(A)$ be such that $M_{\mathfrak{p}} \in G\left(A_{\mathfrak{p}}\right)$. Then there exists $s \in A \backslash \mathfrak{p}$ such that $s H_{\mathfrak{a}}^{i}(M)=0$ for all $i<\operatorname{grade}(\mathfrak{a}, A)$.

Proof. We use an inductive argument on $i$. Let $M$ be a finitely generated $A$-module, and let $\mathfrak{p} \in \operatorname{Spec}(A)$ be such that $M_{\mathfrak{p}} \in G\left(A_{\mathfrak{p}}\right)$. If $i=0$, since $H_{\mathfrak{a}}^{0}(M)$ is a finitely generated $A$-module and, in view of $2.8, \mathfrak{p} \notin \operatorname{Supp} H_{\mathfrak{a}}^{0}(M)$, there exists $s \in A \backslash \mathfrak{p}$ such that $s H_{\mathfrak{a}}^{0}(M)=0$. Now suppose that $1 \leq i<\operatorname{grade}(\mathfrak{a}, A)$ and that the result holds for smaller values of $i$. Consider the exact sequence

$$
0 \longrightarrow K \longrightarrow M \stackrel{\delta_{M}}{\longrightarrow} M^{* *} \longrightarrow L \longrightarrow 0
$$

where $M^{* *}$ is the bidual of $M$. Since the $A$-modules $K$ and $L$ are finitely generated and $\mathfrak{p} \notin \operatorname{Supp}(K) \cup \operatorname{Supp}(L)$, there exists $t \in A \backslash \mathfrak{p}$ such that $t H_{\mathfrak{a}}^{i}(K)=0=$ $t H_{\mathfrak{a}}^{i}(L)$ for all $i \geq 0$. Hence, by breaking the above exact sequence into short exact sequences, we need only to prove the result for $M^{* *}$. Note that, by [4, 1.1.7], $M_{\mathfrak{p}}^{* *} \in G\left(A_{\mathfrak{p}}\right)$. Now, let

$$
0 \longrightarrow W \longrightarrow F \longrightarrow M^{*} \longrightarrow 0
$$

be a finite presentation of $M^{*}$. By applying the duality functor $-{ }^{*}=\operatorname{Hom}_{A}(-, A)$, we obtain the exact sequence

$$
0 \longrightarrow M^{* *} \longrightarrow F \longrightarrow W^{*} \longrightarrow \operatorname{Ext}_{A}^{1}\left(M^{*}, A\right) \longrightarrow 0,
$$

which breaks up into two short exact sequences

$$
0 \longrightarrow M^{* *} \longrightarrow F \longrightarrow D \longrightarrow 0 \text { and } 0 \longrightarrow D \longrightarrow W^{*} \longrightarrow \operatorname{Ext}_{A}^{1}\left(M^{*}, A\right) \longrightarrow 0 \text {. }
$$

Since grade $(\mathfrak{a}, A)>i$, we get from the induced long exact sequence of $H_{\mathfrak{a}}^{i}$,

(1) $H_{\mathfrak{a}}^{i}\left(M^{* *}\right) \cong H_{\mathfrak{a}}^{i-1}(D)$, and

(2) $H_{\mathfrak{a}}^{i-2}\left(\operatorname{Ext}_{A}^{1}\left(M^{*}, A\right)\right) \longrightarrow H_{\mathfrak{a}}^{i-1}(D) \longrightarrow H_{\mathfrak{a}}^{i-1}\left(W^{*}\right)$ is exact.

On the other hand, since $\operatorname{Ext}_{A}^{1}\left(M^{*}, A\right)$ is a finitely generated $A$-module and $\mathfrak{p} \notin \operatorname{Supp}\left(\operatorname{Ext}_{A}^{1}\left(M^{*}, A\right)\right)$, there exists $s \in A \backslash \mathfrak{p}$ such that $s H_{\mathfrak{a}}^{i}\left(\operatorname{Ext}_{A}^{1}\left(M^{*}, A\right)\right)=0$ for all $i \in \mathbb{N}_{0}$ Also, by [4, 1.1.10(a)], we have that $W_{\mathfrak{p}}^{*} \in G\left(A_{\mathfrak{p}}\right)$. Now the conclusion follows by applying the inductive hypotheses on $W^{*}$ in conjunction with (1) and (2).

Theorem 2.10. The Local-global Principle (for the annihilation of local cohomology modules) holds over a commutative Noetherian Gorenstein ring.

Remark 2.11. Whenever $A$ is the homomorphic image of a Gorenstein ring and $M$ a finitely generated $A$-module then, by [2, 3.4(i)] and Faltings' Annihilator Theorem 
(in the local case), for every choice of the ideals $\mathfrak{b}, \mathfrak{a}$ of $A, \lambda_{\mathfrak{a}}^{\mathfrak{b}}(M)=\inf \left\{f_{\mathfrak{a}_{\mathfrak{p}}}^{\mathfrak{b}_{\mathfrak{p}}}\left(M_{\mathfrak{p}}\right) \mid\right.$ $\mathfrak{p} \in \operatorname{Supp}(M)\}$.

Proof of 2.10. We suppose, for ideals $\mathfrak{a}$ and $\mathfrak{b}$ of a Gorenstein ring $A$ and a finitely generated $A$-module $M$, that $f_{\mathfrak{a}}^{\mathfrak{b}}(M)<f_{\mathfrak{a}_{A_{\mathfrak{p}}}}^{\mathfrak{b}_{A_{\mathfrak{p}}}}\left(M_{\mathfrak{p}}\right)$ for all $\mathfrak{p} \in \operatorname{Spec}(A)$ and look for a contradiction. We can (and do) assume that $\mathfrak{a}$ is a maximal member of the set $\{\mathfrak{c}: \mathfrak{c}$ is an ideal of $A$ such that, for some finitely generated $A$-module $M^{\prime}, f_{\mathfrak{c}}^{\mathfrak{b}}\left(M^{\prime}\right)<f_{\mathfrak{c}_{A_{\mathfrak{p}}}}^{\mathfrak{b}_{A_{\mathfrak{p}}}}\left(M_{\mathfrak{p}}^{\prime}\right)$ for all $\left.\mathfrak{p} \in \operatorname{Spec}(A)\right\}$. By [2, 3.2], it follows that $\mathfrak{a}$ is a prime ideal of $A$.

By 2.1 and 2.3, we can assume, in our search for a contradiction, that $\mathfrak{b} \subseteq \mathfrak{a}$, and $0: M / \Gamma_{\mathfrak{b}}(M) \subseteq \mathfrak{a}$. Set $s:=\inf \left\{f_{\mathfrak{a}_{A_{\mathfrak{p}}}}^{\mathfrak{b}_{A_{\mathfrak{p}}}}\left(M_{\mathfrak{p}}\right): \mathfrak{p} \in \operatorname{Supp}(M)\right\}$. By $2.2, s \leq$ ht $\mathfrak{a}$. There are two cases to consider.

Case 1. Suppose that ht $\mathfrak{a}=s$. Let $\mathfrak{p}$ be a prime ideal of $A$ such that $G-$ $\operatorname{dim}_{A_{\mathfrak{p}}} M_{\mathfrak{p}}=0$. Then, by 2.9 , there exists $s_{\mathfrak{p}} \in A \backslash \mathfrak{p}$ such that $s_{\mathfrak{p}} H_{\mathfrak{a}}^{i}(M)=0$ for all $i<s$. Set

$$
S:=\left\{\mathfrak{q} \in \operatorname{Spec}(A): M_{\mathfrak{q}} \in G\left(A_{\mathfrak{q}}\right)\right\},
$$

and let $g$ be the ideal generated by all $s_{\mathfrak{p}}$ 's, where $\mathfrak{p}$ ranges in $S$. In case $S=\emptyset$, we set $g:=0$. Observe that if $g \subseteq \mathfrak{p}$, then $\mathfrak{p} \notin S$. Consequently, $G-\operatorname{dim}_{A_{\mathfrak{p}}} M_{\mathfrak{p}}>0$. Also note that $g H_{\mathfrak{a}}^{i}(M)=0$ for all $i<s$. If $\mathfrak{b} \subseteq \operatorname{Rad}(g)$, then we have nothing to do any more. So we may assume that $\mathfrak{b} \nsubseteq \operatorname{Rad}(g)$. Let $\mathfrak{p}_{1}, \ldots, \mathfrak{p}_{t}$ be the minimal primes over $g$ not containing $\mathfrak{b}$. It is easy to see that $\bigcap_{i=1}^{t} \mathfrak{p}_{i} \nsubseteq \mathfrak{a}$. (If $\mathfrak{p}_{i} \subset \mathfrak{a}$, by the same argument as in [3, 9.4.8], one can deduce that $G-\operatorname{dim} M_{\mathfrak{p}_{i}}>0$ and so, by the Auslander-Bridger formula (cf. [4, 1.4.8]), $\operatorname{depth} R_{\mathfrak{p}_{i}}-\operatorname{depth} M_{\mathfrak{p}_{i}}>0$. This implies that $\lambda_{\mathfrak{a}}^{\mathfrak{b}}(M) \leq \operatorname{depth} M_{\mathfrak{p}_{i}}+\operatorname{ht} \frac{\mathfrak{a}+\mathfrak{p}_{i}}{\mathfrak{p}_{i}}<\operatorname{depth} R_{\mathfrak{p}_{i}}+$ ht $\frac{\mathfrak{a}}{\mathfrak{p}_{i}} \leq$ ht $\mathfrak{a}$, which is a contradiction.) Let $x \in \bigcap_{i=1}^{t} \mathfrak{p}_{i} \backslash \mathfrak{a}$. Note that $\mathfrak{b}_{x} \subseteq(\operatorname{Rad}(g))_{x}$. Now it follows from the exact sequence

$$
\cdots \longrightarrow H_{\mathfrak{a}+A x}^{i}(M) \longrightarrow H_{\mathfrak{a}}^{i}(M) \longrightarrow H_{\mathfrak{a}_{x}}^{i}\left(M_{x}\right) \longrightarrow \cdots
$$

that $f_{(\mathfrak{a}+A x) A_{\mathfrak{p}}}^{\mathfrak{b}_{A_{\mathfrak{p}}}}\left(M_{\mathfrak{p}}\right) \geq s$. Thus by the "maximality" assumption about $\mathfrak{a}$, we have that $f_{\mathfrak{a}+A x}^{\mathfrak{b}}(M) \geq s$. Now, we deduce from the above exact sequence that $f_{\mathfrak{a}}^{\mathfrak{b}}(M) \geq$ $s$.

Case 2. Suppose that ht $\mathfrak{a}>s$. Consider the finite presentation

$$
0 \longrightarrow K \longrightarrow F \longrightarrow M \longrightarrow 0
$$

of $M$ to deduce the long exact sequence

$$
\cdots \longrightarrow H_{\mathfrak{a}}^{i}(M) \longrightarrow H_{\mathfrak{a}}^{i+1}(K) \longrightarrow H_{\mathfrak{a}}^{i+1}(F) \longrightarrow \cdots .
$$

Therefore, $f_{\mathfrak{a} A_{\mathfrak{p}}}^{\mathfrak{b} A_{\mathfrak{p}}}\left(K_{\mathfrak{p}}\right)>s$ for all $\mathfrak{p} \in \operatorname{Spec}(A)$. Hence, by case $1, f_{\mathfrak{a}}^{\mathfrak{b}}(K) \geq s+1$. Now, by using the exact sequence $(*)$, we can deduce that $f_{\mathfrak{a}}^{\mathfrak{b}}(M) \geq s$.

Corollary 2.12. The Annihilator Theorem (for local cohomology modules) holds over a commutative Noetherian Gorenstein ring.

Proof. Let $A$ be a Gorenstein ring, let $\mathfrak{a}$ and $\mathfrak{b}$ be ideals of $A$, and let $M$ be a finitely generated $A$-module. We must show that $f_{\mathfrak{a}}^{\mathfrak{b}}(M)=\lambda_{\mathfrak{a}}^{\mathfrak{b}}(M)$. By [2, 3.7], we may assume that $\mathfrak{b} \subseteq \mathfrak{a}$. Hence, by [3, 9.3.5], the only nontrivial point is the 
proof that $\lambda_{\mathfrak{a}}^{\mathfrak{b}}(M) \leq f_{\mathfrak{a}}^{\mathfrak{b}}(M)$. By 2.10 , there exists a prime ideal $\mathfrak{p}$ of $A$ such that $f_{\mathfrak{a}}^{\mathfrak{b}}(M)=f_{\mathfrak{a} A_{\mathfrak{p}}}^{\mathfrak{b} A_{\mathfrak{p}}}\left(M_{\mathfrak{p}}\right)$. Also, by [5], $f_{\mathfrak{a} A_{\mathfrak{p}}}^{\mathfrak{b} A_{\mathfrak{p}}}\left(M_{\mathfrak{p}}\right)=\lambda_{\mathfrak{a} A_{\mathfrak{p}}}^{\mathfrak{b} A_{\mathfrak{p}}}\left(M_{\mathfrak{p}}\right)$. We now use [3] 9.2.5] to complete the proof.

Corollary 2.13. The Local-global Principle and the Annihilator Theorem (for local cohomology modules) hold over a homomorphic image of a commutative Noetherian Gorenstein ring.

\section{ACKNOWLEDGMENTS}

The authors are deeply grateful to the anonymous referee for his or her useful pointed comments on the paper and for drawing the authors' attention to Remark 2.11 .

\section{REFERENCES}

[1] M. Brodmann, Einige Ergebnisse aus der lokalen Kohomologietheorie und ihre Anwendung, Osnabrücker Schriften zur Mathematik 5 (1983). MR 87j:14005

[2] M. Brodmann, Ch. Rotthaus, and R. Y. Sharp, On annihilators and associated primes of local cohomology modules, J. Pure Appl. Algebra, 153 (2000) 197-227. MR 2002b:13027

[3] M. Brodmann and R. Y. Sharp, Local cohomology: an algebraic introduction with geometric applications, Cambridge Studies in Advanced Mathematics, No. 60, Cambridge University Press, Cambridge, 1998. MR 99h:13020

[4] L. W. Christensen, Gorenstein dimensions, Lecture Notes in Mathematics, no. 1747, Springer-Verlag, Berlin, 2000. MR 2002e:13032

[5] G. Faltings, Über die Annulatoren lokaler Kohomologiegruppen, Arch. Math. (Basel) 30 (1978), 473-476. MR 58:22058

[6] G. Faltings, Der Endlichkeitssatz in der lokalen Kohomologie, Math. Ann. 255 (1981), 45-56. MR 82f:13003

[7] K. N. Raghavan, Local-global principle for annihilation of local cohomology, Contemporary Math. 159 (1994), 329-331. MR 95c:13018

[8] K. N. Raghavan, Uniform annihilation of local cohomology and of Koszul homology, Math. Proc. Cambridge Philos. Soc. 112 (1992), 487-494. MR 94e:13033

Institute for Studies in Theoretical Physics and Mathematics, P.O. Box 19395-5746, Tehran, Iran - and - Department of Mathematics, Damghan University, P.O. Box 36715364, DAMGHAN, IRAN

E-mail address: Khashyar@ipm.ir

Institute for Studies in Theoretical Physics and Mathematics, P.O. Box 19395-5746, Tehran, Iran - and - Department of Mathematics, Damghan University, P.O. Box 36715364, DAMGHAN, Iran

E-mail address: Salarian@ipm.ir 